\title{
Candida albicans sphingolipid C9- methyltransferase is involved in hyphal elongation
}

\author{
Takahiro Oura and Susumu Kajiwara
}

Correspondence

Susumu Kajiwara

skajiwar@bio.titech.ac.jp

Received 28 September 2009

Revised 27 November 2009

Accepted 16 December 2009
Department of Life Science, Graduate School of Bioscience and Biotechnology, Tokyo Institute of
Technology, 4259-B5 Nagatsuta, Midori-ku, Yokohama, Kanagawa 266-8501, Japan

C9-methylated glucosylceramide is a fungus-specific sphingolipid. This lipid is a major membrane component in the cell and is thought to play important roles in the growth and virulence of several fungal species. To investigate the importance of the methyl branch of the long-chain base in glucosylceramides in pathogenic fungi, we identified and characterized a sphingolipid C9-methyltransferase gene (MTS1, C9-MethylTransferase for Sphingolipid 1) in the pathogenic yeast Candida albicans. The $m t s 1$ disruptant lacked $(E, E)-9$-methylsphinga-4,8-dienine in its glucosylceramides and contained $(E)$-sphing-4-enine and $(E, E)$-sphinga-4,8-dienine.

Reintroducing the MTS1 gene into the $m t s 1$ disruptant restored the synthesis of $(E, E)-9$ methylsphinga-4,8-dienine in the glucosylceramides. We also created a disruptant of the HSX11 gene, encoding glucosylceramide synthase, which catalyses the final step of glucosylceramide synthesis, in C. albicans and compared this mutant with the $m t s 1$ disruptant. The C. albicans $m t s 1$ and $h s \times 11$ disruptants both had a decreased hyphal growth rate compared to the wild-type strain. The $h s \times 11$ disruptant showed increased susceptibility to SDS and fluconazole, similar to a previously reported s/d1 disruptant that contained only $(E)$-sphing-4-enine in its glucosylceramides, suggesting that these strains have defects in their cell membrane structures. In contrast, the mts1 disruptant grew similarly to wild-type in medium containing SDS or fluconazole. These results suggest that the C9-methyl group of a long-chain base in glucosylceramides plays an important role in the hyphal elongation of C. albicans independent of lipid membrane disruption.

\section{INTRODUCTION}

Glycosphingolipids, which consist of a ceramide with various sugars, are ubiquitous membrane lipids in eukaryotic organisms. They are classified by their characteristic polar headgroups. One type of glycosphingolipid, glycosylinositolphosphorylceramide (GIPC), is linked with inositol via a phosphodiester bond and is present only in fungi and plants. On the other hand, glycosylceramides (GlyCers), which are directly linked to a glucosyl or galactosyl residue, can form the base for further glycosylations. Glucosylceramides (GluCers), which contain a directly linked glucose residue, are unique glycosphingolipids that are found in animals, plants and fungi, whereas galactosylceramides, which are linked with galactose, are found only in animals and fungi (reviewed by Warnecke \& Heinz, 2003).

In animals, GlyCers are not only essential for growth but are also thought to play a crucial role in cell differentiation and cell-cell interactions (reviewed by Merrill et al., 1997; Ternes et al., 2002). Plant GlyCers and their components such as ceramide and long-chain bases (LCBs) have been

Abbreviations: CFW, Calcofluor white; GluCer, glucosylceramide; GlyCer, glycosylceramide; LCB, long-chain base. suggested to function in signal transduction and hostpathogen interactions (reviewed by Sperling \& Heinz, 2003; Thevissen et al., 2004; Ramamoorthy et al., 2007). Although two yeast species, Saccharomyces cerevisiae and Schizosaccharomyces pombe, have been used as models to study the metabolism and function of sphingolipids in higher eukaryotic cells, these species are not capable of synthesizing GlyCers. Therefore, our understanding of the molecular mechanisms that contribute to the function of GlyCer in fungi is still limited.

Recently, a number of fungal pathogens have been reported to contain GluCers that play functional roles in growth and differentiation. The addition of antibodies against a purified fungal GluCer inhibited cell budding and growth in Cryptococcus neoformans, and antibodies against a purified Pseudallescheria boydii GluCer inhibited mycelium formation in P. boydii and germ-tube formation in Candida albicans (Rodrigues et al., 2000; Pinto et al., 2002). Inhibiting glucosylceramide synthase, which catalyses the final step in GluCer synthesis, influences the growth and differentiation of Aspergillus nidulans and Aspergillus fumigatus (Levery et al., 2002). Moreover, additional evidence has emerged that GluCers play important roles in 
fungal pathogenicity. A $\Delta g c s 1$ strain of Cr. neoformans, which lacks GluCer, is avirulent, and the virulence of a Fusarium graminearum $\Delta g c s 1(\Delta \mathrm{Fggcs} 1)$ strain in wheat is highly compromised (Ramamoorthy et al., 2007). However, it is still unknown what part(s) of the GluCer molecular structures cause(s) these biological phenomena in fungi.

GluCer biosynthesis starts with the condensation of palmitoyl-CoA and serine by serine palmitoyltransferase to form 3-ketosphinganine (reviewed by Warnecke \& Heinz, 2003). 3-Ketosphinganine reductase catalyses the conversion of 3-ketosphinganine to sphinganine. Ceramide synthase catalyses the condensation of sphinganine to saturated acyl-CoA for the synthesis of ceramides. Next, sphingolipid $\Delta^{4}$-desaturase catalyses the $\Delta^{4}$-desaturation of LCB in ceramides (Ternes et al., 2002). In many plants and fungi, sphingolipid $\Delta^{8}$-desaturase catalyses the $\Delta^{8-}$ desaturation of LCB in ceramides, while sphingolipid C9-methyltransferase introduces a methyl group at C9 of LCB in ceramides (Sperling et al., 1998; Takakuwa et al., 2002; Ternes et al., 2006). The desaturation of fatty acyl groups in ceramides has also been detected in some organisms (reviewed by Warnecke \& Heinz, 2003). Finally, these ceramides react with UDP-glucose, catalysed by glucosylceramide synthase, resulting in GluCer (Leipelt et al., 2001) (Fig. 1). Since it has been suggested that Sac. cerevisiae and Sch. pombe lack most of the genes that encode the enzymes for GluCer synthesis, there is currently little knowledge about the fungal genes that are involved in GluCer synthesis. It was recently reported that a $C r$. neoformans strain that lacks the GluCer synthase gene and cannot synthesize GluCer was incapable of growing in neutral and alkaline solutions in vitro, which reduces its pathogenicity in its host (Rittershaus et al., 2006). In contrast, disruption of the GluCer synthase gene (HSX11) in C. albicans does not affect the growth of its unicellular or filamentous forms (Leipelt et al., 2001).

Previously, we characterized the sphingolipid $\Delta^{8}$-desaturase gene (SLD1) from C. albicans and constructed a disruptant of this gene. Compared to wild-type, the C. albicans sld1 disruptant had a decreased hyphal growth rate and was highly sensitive to SDS and fluconazole (Oura \& Kajiwara, 2008). Therefore, it was suggested that $(E, E)$-sphinga-4,8dienine and/or (E,E)-9-methylsphinga-4,8-dienine in ceramide and GluCer (Fig. 1) play a role in the morphogenesis of this fungus. More recently, two genes encoding a putative sphingolipid C9-methyltransferase in F. graminearum (named FgMT1 and FgMT2) were characterized (Ramamoorthy et al., 2009). Although the $\Delta \mathrm{Fg} m t 1$ mutant was able to produce GluCers, including (E,E)-9-methylsphinga-4,8-dienine (C9-methylated GluCer), similarly to the wild-type, the mutant had a reduced amount of this GluCer compared to the wild-type. The $\Delta \mathrm{Fg} m t 2$ mutant also exhibited a severe growth defect, produced abnormal conidia, and showed reduced disease symptoms in wheat and much delayed disease symptoms in Arabidopsis thaliana. These results suggested that C9-methylated GluCers play a functional role in the growth, differenti-

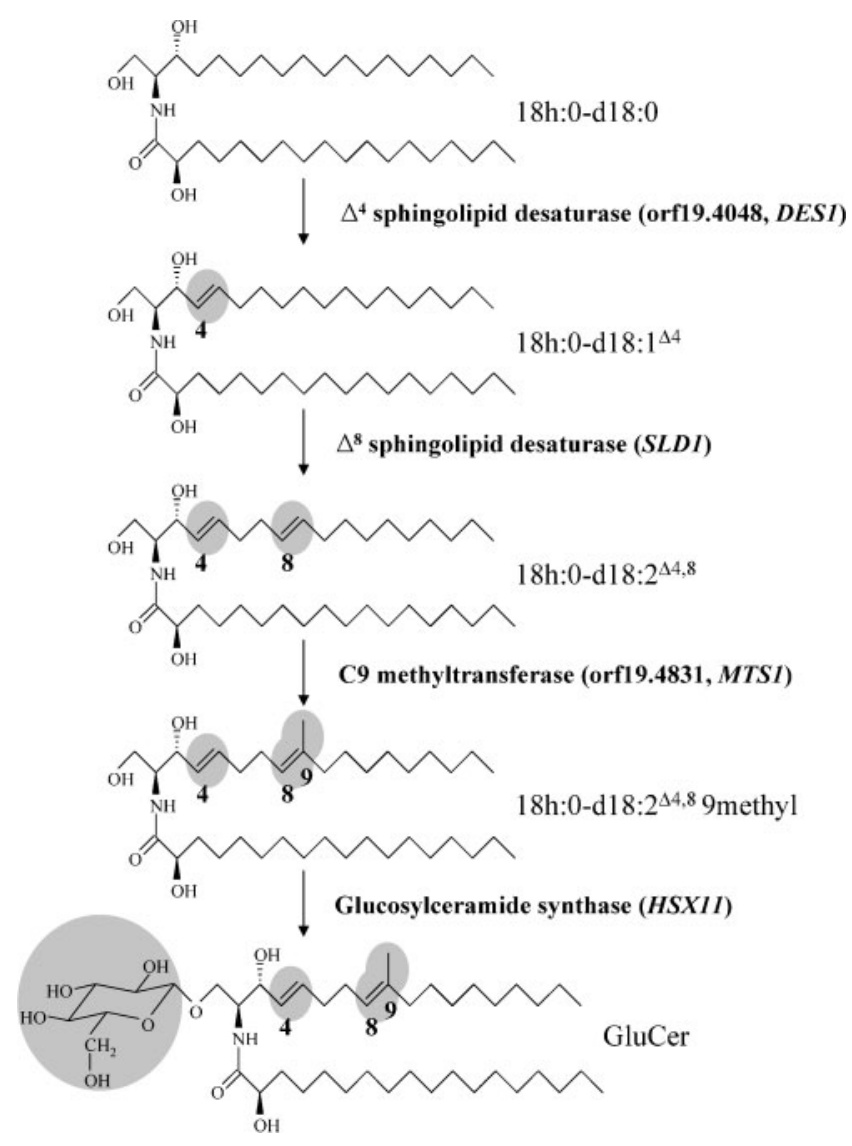

Fig. 1. Biosynthetic pathway of glucosylceramide in C. albicans. Gene symbols are according to the C. albicans nomenclature in the Candida Genome Database and are shown in italic.

ation and virulence of this fungus. Therefore, the structure of the LCB portion of GluCer was suggested to be responsible for the determination of biological phenomena in pathogenic fungi.

In this study, we focused on two other genes involved in GluCer biosynthesis, the MTS1 and HSX11 genes in C. albicans. We constructed a null mutant of MTS1 to determine the function of this gene in C. albicans, and to investigate the effects of MTS1 disruption on the morphogenesis of this fungus. We also constructed a hsx11 null mutant of $C$. albicans and compared the phenotype of this mutant with that of the mts1 null mutant. Both the mtsl and $h s \times 11$ mutants had an obvious delay in filamentous growth compared to the isogenic wild-type strains. The mutants were also found to differ in their susceptibility to some antifungal drugs and environmental stressors.

\section{METHODS}

Media, growth conditions, and basic techniques. The C. albicans strains used and constructed in this study are summarized in Table 1. 
Strains were typically grown at $30{ }^{\circ} \mathrm{C}$ in YPD medium $[1 \%$ Bacto yeast extract, $2 \%$ Bacto peptone, $2 \%$ dextrose (glucose) ( $\mathrm{pH} 5.6$ )] or complete minimal (CM) medium (Ausubel et al., 1992) without uracil and/or arginine. The rate of growth was measured as $\mathrm{OD}_{600}$ using an Ultrospec 3000 spectrophotometer (Pharmacia Biotech). The yeast-tohypha transition was induced at $37^{\circ} \mathrm{C}$ in both solid and liquid media. For the solid transition medium, we used $10 \%$ fetal bovine serum medium (Hanaoka et al., 2005). For the liquid transition medium, we used $10 \%$ fetal bovine serum in YPD at $\mathrm{pH}$ 7.2. Filamentation in the liquid medium was induced by inoculating $2 \times 10^{6}-5 \times 10^{6} \mathrm{cells} \mathrm{ml}^{-1}$ and incubating at $37{ }^{\circ} \mathrm{C}$. For spot tests, cells were grown overnight at $30{ }^{\circ} \mathrm{C}$ in YPD medium and then tenfold serial dilutions were spotted onto YPD agar plates and grown for $1-2$ days at $30{ }^{\circ} \mathrm{C}$. The antifungals used in this study were amphotericin B (Wako Pure Chemical Industries), fluconazole (LKT Laboratories), micafungin (Astellas Pharma) and terbinafine (LKT Laboratories).

Escherichia coli $\mathrm{DH} 5 \alpha$ and the cloning vector pBluescript II SK+ (Stratagene) were used to manipulate the DNA. General recombinant DNA procedures were performed as described by Sambrook et al. (1989). C. albicans was transformed following the method described by Umeyama et al. (2005).

Plasmid construction. The primers used in this study are listed in Table 2. The MTS1 DNA fragment was PCR amplified using primers mts1-clo5 and mts1-clo3, with TUA4 chromosomal DNA as a template. The PCR products were cloned into the EcoRV site of pBluescript II SK + to generate pBS-MTS1. The nucleotide sequence of the MTS1 DNA was confirmed by DNA sequencing. The $1.5 \mathrm{~kb}$ BamHI-XhoI DNA fragment from pBS-MTS1 was inserted into the BamHI-XhoI sites of pFLAG-Actl to generate pFLAG-Act1-MTS1. The HSX11 DNA fragment was PCR amplified using primers hsx11clo5 and hsx11-clo3, with TUA4 chromosomal DNA as a template. These products were cloned into the EcoRV site of pBluescript II SK+ to generate pBS-HSX11. The nucleotide sequence of this fragment was confirmed. The $1.6 \mathrm{~kb} \mathrm{BamHI-XhoI} \mathrm{DNA} \mathrm{fragment} \mathrm{from} \mathrm{pBS-}$
HSX11 was inserted into the BamHI-XhoI sites of pFLAG-Act1 (Umeyama et al., 2002) to generate pFLAG-Act1-HSX11.

Disruption of $\mathbf{M T S 1}$ in $\boldsymbol{C}$. albicans and construction of a $\boldsymbol{m t s} \mathbf{1}$
mutant expressing MTS1. To disrupt MTS1 in a diploid C. albicans strain, two markers were used. The $0.5 \mathrm{~kb}$ DNA fragment corresponding to the 3' end of MTS1 was PCR amplified using primers mts1-mutNot5 and mts1-mut-Sac3, digested with NotI and SacI, and then cloned into the NotI-SacI sites of pBS-hph200-URA3 and pUC19-ARG4 to generate pBS-hph200-URA3-NS and pUC-ARG4-NS, respectively. The $0.5 \mathrm{~kb}$ DNA fragment corresponding to the $5^{\prime}$ end of MTS1 was PCR amplified using primers mts1-mut-Kpn5 and mts1-mut-Xho3, digested with $K p n I$ and $X h o I$, and then cloned into the KpnI-XhoI sites of pBS-hph200-URA3-NS to generate pBS-hph200-URA3-disMTS1. The $0.5 \mathrm{~kb}$ DNA fragment corresponding to the $5^{\prime}$ end of MTS1 was PCR amplified using primers mts1-mut-Sph5 and mts1-mut-Kpn3, digested with $S p h \mathrm{I}$ and $K p n \mathrm{I}$, and then cloned into the SphI-KpnI sites of pUC-ARG4-NS to generate pUC-ARG4-disMTS1.

The $3.1 \mathrm{~kb} K p n \mathrm{I}-\mathrm{SacI}$ fragment of pBS-hph200-URA3-disMTS1 was used to transform the C. albicans $\mathrm{Arg}^{-} \mathrm{Ura}^{-}$strain TUA4 to generate a $\mathrm{Ura}^{+}$transformant (MTS101). Then the $3.1 \mathrm{~kb}$ SphI-SacI fragment of pUC-ARG4-disMTS1 was used to transform the MTS101 strain $\left(\mathrm{Arg}^{-}\right)$to generate MTS102. The resulting $\mathrm{Ura}^{+}$ $\mathrm{Arg}^{+}$transformant MTS102 was plated on medium containing 5fluoroorotic acid to isolate the $\mathrm{Ura}^{-}$segregant (MTS103). To confirm gene disruption, genomic DNA was isolated from each strain, digested with EcoRV, run on a $1 \%$ agarose gel, and then transferred onto a Hybond-N+ nylon membrane (Amersham Biosciences). Southern hybridization was performed using a ${ }^{32} \mathrm{P}$ labelled probe in a solution containing $5 \times$ SSC, $6 \times$ Denhart's solution and $0.1 \mathrm{mg}$ herring sperm DNA ml ${ }^{-1}$ at $65{ }^{\circ} \mathrm{C}$ according to the methods of Sambrook et al. (1989). The MTS1 gene was reintroduced into the $C a-R P 10$ locus of the null mutant by using AatI-digested pFLAG-Act1-MTS1 to transform strain MTS103,

Table 1. Yeast strains generated and tested in this study

\begin{tabular}{|c|c|c|}
\hline Strain & Genotype & Reference \\
\hline TUA4 & 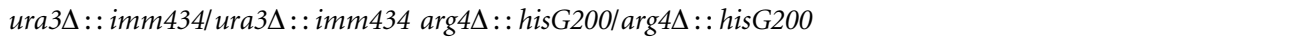 & NIID $^{*}$ \\
\hline TUA6 & 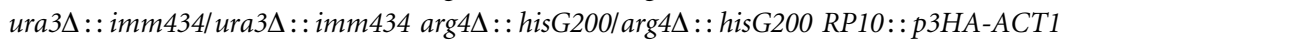 & $\mathrm{NIID}^{*}$ \\
\hline MTS101 & 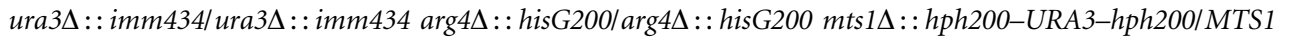 & This work \\
\hline MTS102 & 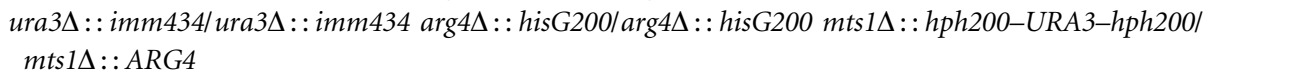 & This work \\
\hline MTS103 & 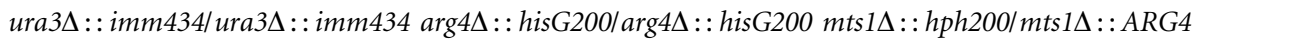 & This work \\
\hline MTS104 & 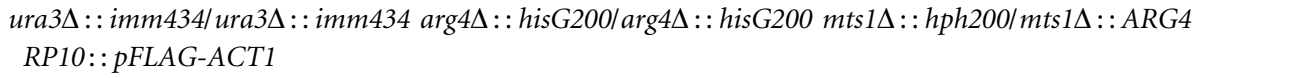 & This work \\
\hline MTS105 & 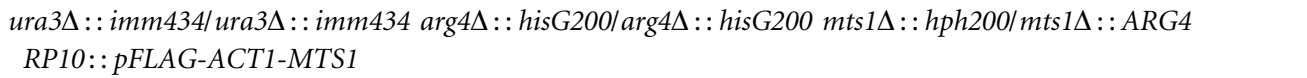 & This work \\
\hline HSX101 & 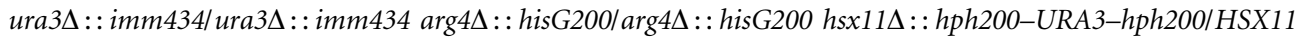 & This work \\
\hline HSX102 & 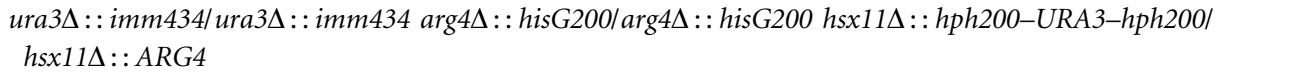 & This work \\
\hline HSX103 & 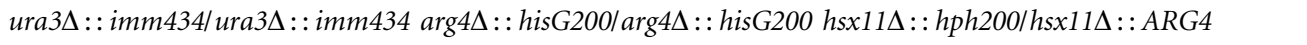 & This work \\
\hline HSX104 & 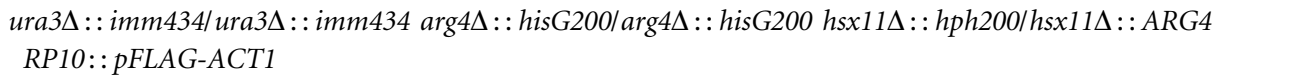 & This work \\
\hline HSX105 & 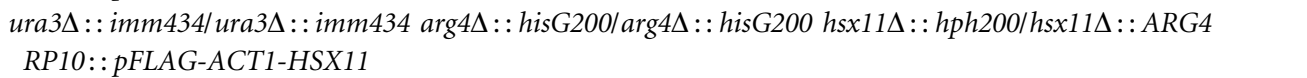 & This work \\
\hline
\end{tabular}

${ }^{*}$ NIID, National Institute of Infectious Diseases, Japan. 
Table 2. Oligonucleotide primers used in this study

Restriction enzyme sites are underlined.

\begin{tabular}{|c|c|}
\hline Oligonucleotide & Sequence $\left(5^{\prime}-3^{\prime}\right)$ \\
\hline mts1-clo5 & AAGGATCCAGGATGTTAGACGACGTTGCATTCATCAAG -3' \\
\hline mts1-clo3 & 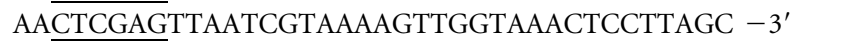 \\
\hline mts1-mut-Sph5 & AAGCATGCCCAACACCAATCAATTGACTTTCG - $3^{\prime}$ \\
\hline mts1-mut-Kpn3 & 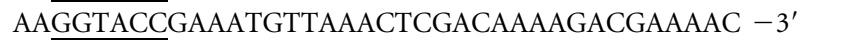 \\
\hline mts1-mut-Not5 & AAGCGGCCGCACTGGTTGAGCTTTTATGGTGTATTTG -3' \\
\hline mts1-mut-Sac3 & 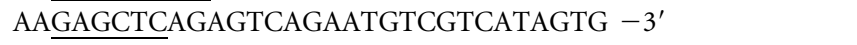 \\
\hline mts1-mut-Kpn5 & AAGGTACCCAACACCAATCAATTGACTTTC - $3^{\prime}$ \\
\hline mts1-mut-Xho3 & AACTCGAGAAATGTTAAACTCGACAAAAGACGAAAAC - -3' \\
\hline hsx11-clo5 & AAGGATCCAGGATGGTTCAAGAAGAATTATCCTTATTTAGG -3' \\
\hline hsx11-clo3 & AACTCGAGTCACATTTCTTCAGCAGTTAAATCTTTCTTAATC -3' \\
\hline hsx11-mut-Bam5 & AAGGATCCAGTAATGGCACCTTATTGTAATGTATG - ${ }^{\prime}$ \\
\hline hsx11-mut-Kpn3 & AAGGTACCGAAGAACAAAAGATTACGATATTTCACAGTG -3' \\
\hline hsx11-mut-Not5 & AAGCGGCCGCTACGCCAATTGTTATAGATAGATAGACAGG -3' \\
\hline hsx11-mut-Sac3 & AAGAGCTCGCAATATCAGGATCATAATGTTTATACCATG - 3' \\
\hline hsx11-mut-Kpn5 & AAGGTACCAGTAATGGCACCTTATTGTAATGTATG - $3^{\prime}$ \\
\hline hsx11-mut-Xho3 & AACTCGAGAAGAACAAAAGATTACGATATTTCACAGTG -3' \\
\hline
\end{tabular}

generating MTS105. The AatI-digested empty vector pFLAG-Act1 was integrated into strain MTS103 to generate MTS104 as a control.

\begin{abstract}
Disruption of HSX11 in C. albicans and construction of a hsX11 strain expressing HSX11. To disrupt HSX11 in C. albicans, the $A R G 4$ and URA3 genes were used as markers. The $0.6 \mathrm{~kb}$ DNA fragment corresponding to the $3^{\prime}$ end of HSX11 was PCR amplified using primers hsx11-mut-Not5 and hsx11-mut-Sac3, digested with NotI and SacI, and then cloned into the NotI-SacI sites of pBShph200-URA3 (Oura \& Kajiwara, 2008) and pUC19-ARG4 (Hanaoka et al., 2005) to generate pBS-hph200-URA3-NS and pUC-ARG4-NS, respectively. The $0.6 \mathrm{~kb}$ DNA fragment corresponding to the $5^{\prime}$ end of HSX11 was PCR amplified using primers hsx11-mut-Kpn5 and hsx11-mut-Xho3, digested with $K p n I$ and $X h o I$, and then cloned into the KpnI-XhoI sites of pBS-hph200-URA3-NS to generate pBShph200-URA3-disHSX11. The $0.6 \mathrm{~kb}$ DNA fragment corresponding to the $5^{\prime}$ end of HSX11 was PCR amplified using primers hsx11-mutBam5 and hsx11-mut-Kpn3, digested with BamHI and KpnI, and then cloned into the BamHI-KpnI sites of pUC-ARG4-NS to generate pUC-ARG4-disHSX11.
\end{abstract}

The $3.2 \mathrm{~kb}$ KpnI-SacI fragment of pBS-hph200-URA3-disHSX11 was used to transform the C. albicans $\mathrm{Arg}^{-} \mathrm{Ura}^{-}$strain TUA4 to generate the $\mathrm{Ura}^{+}$transformant HSX101. Then the $3.2 \mathrm{~kb}$ BamHI-SacI fragment of pUC-ARG4-disHSX11 was used to transform HSX101 $\left(\mathrm{Arg}^{-}\right)$to generate HSX102. The resulting $\mathrm{Ura}^{+} \mathrm{Arg}^{+}$transformant, HSX102, was plated on a medium containing 5-fluoroorotic acid to isolate the $\mathrm{Ura}^{-}$segregant (HSX103). To confirm gene disruption, genomic DNA was isolated from each strain, digested with SpeI, run on a $1 \%$ agarose gel, and then transferred onto a Hybond-N + nylon membrane (Amersham Biosciences). Southern hybridization was performed using a ${ }^{32} \mathrm{P}$-labelled probe in a solution containing $5 \times$ SSC, $6 \times$ Denhart's solution, and $0.1 \mathrm{mg}$ herring sperm DNA ml ${ }^{-1}$ at $65{ }^{\circ} \mathrm{C}$ according to the methods of Sambrook et al. (1989). The HSX11 gene was reintroduced into the RP10 locus of the null mutant by using AatI-digested pFLAG-Act1-HSX11 to transform strain HSX103, generating HSX105. The AatI-digested empty pFLAG-Act1 vector was integrated into strain HSX103 to generate HSX104.

Determination of glucosylceramides. GluCers were extracted essentially as described by Saito et al. (2005) with the following modifications. The lyophilized cells $(50 \mathrm{mg})$ were treated with chloroform/methanol $(1: 1, \mathrm{v} / \mathrm{v})$ and $2.0 \mathrm{ml} 0.8 \mathrm{M} \mathrm{KOH} / \mathrm{methanol}$ for 5 min with a subsonic homogenizer (HOM-100, Asahi Grass) and then incubated at $42{ }^{\circ} \mathrm{C}$ for $30 \mathrm{~min}$. Organic salt was collected from the homogenate after mixing with $5.0 \mathrm{ml}$ chloroform and $2.25 \mathrm{ml}$ water, dried using a rotary evaporator, and dissolved in $100 \mu \mathrm{l}$ chloroform/methanol $(1: 1, \mathrm{v} / \mathrm{v})$. Ten microlitres of the extract was spotted onto a TLC plate (silica gel 60, Merck) and the chromatogram was developed using chloroform/methanol/water ( $65: 16: 2$, by vol.) as mobile phase. After development, the plates were sprayed with orcinol/sulfuric acid reagent [ $0.1 \mathrm{~g}$ orcinol in $45 \mathrm{ml}$ of a sulfuric acid/ water/ethanol solution $(5: 13: 27$, by vol.)] and heated on a hotplate to detect the GluCers. GluCers prepared from the mushroom Grifola frondosa were used as standards.

Sphingoid base analysis. Analysis of the sphingoid base composition was performed essentially as described by Tanji et al. (2004) with the following modifications. The lyophilized cells were treated in chloroform/methanol $(2: 1, \mathrm{v} / \mathrm{v})$ for $10 \mathrm{~min}$ with a subsonic homogenizer (HOM-100, Asahi Grass). Then the total lipids were extracted with 4 vols each of chloroform/methanol $(2: 1, \mathrm{v} / \mathrm{v})$ and chloroform/ methanol $(1: 2, \mathrm{v} / \mathrm{v})$ and hydrolysed in $0.4 \mathrm{M} \mathrm{KOH} / \mathrm{methanol}$ at $37^{\circ} \mathrm{C}$ for $2 \mathrm{~h}$. After washing, the organic phase was dried on a rotary evaporator to yield the alkali-stable lipids. GluCers were isolated on preparative TLC plates that were developed in chloroform/methanol $(95: 12, v / v)$ to separate the GluCers from other lipids. The GluCers obtained were degraded with $10 \% \mathrm{Ba}(\mathrm{OH})_{2} /$ dioxane $(1: 1, \mathrm{v} / \mathrm{v})$ and the released sphingoid bases were extracted with diethyl ether and reacted with $0.2 \mathrm{M}$ sodium periodate for $2 \mathrm{~h}$ to obtain the fatty aldehydes. The fatty aldehydes were extracted in dichloromethane and analysed by GLC (GC-18A, Shimadzu) on a $0.25 \mathrm{~mm} \times 50 \mathrm{~m}$ CP-Sil 88 capillary column (Varian). The initial column temperature of $150{ }^{\circ} \mathrm{C}$ was maintained for $2 \mathrm{~min}$, increased by $2{ }^{\circ} \mathrm{C} \mathrm{min}^{-1}$ to $190{ }^{\circ} \mathrm{C}$, and then maintained for $6 \mathrm{~min}$.

\section{RESULTS}

\section{Construction of $\boldsymbol{m t s} 1$ and $h s \times 11$ mutants}

The C. albicans ORF (orf19.4831) encoding a homologue of the sphingolipid C9-methyltransferase of Pichia pastoris was 
previously identified in the C. albicans genome (Ternes et al., 2006). However, this ORF has not been further characterized. To determine whether this ORF (named MTS1) encodes the C. albicans C9-methyltransferase, we constructed $m t s 1$ mutants as described in Methods. For the first allele, MTS1 was replaced with an hph200-URA3hph200 cassette (MTS101) and the remaining allele was replaced with ARG4. The resulting strain was called MTS102. The URA3 gene was excised from MTS102 to generate the Ura-auxotrophic derivative MTS103. Southern blot analysis was performed to confirm that the mutant obtained had a disrupted MTS1 locus (data not shown). Next, to construct the MTS1-recovered strain, an MTS expression plasmid (pFLAG-Act1-MTS1) and its vector (pFLAG-ACT1), which contain the URA3 gene as a selectable marker, were introduced into the RP10 locus (Murad et al., 2000) in the genome of the $m t s 1$ mutant strain MTS103, yielding strains MTS105 and MTS104, respectively.

It was reported that disrupting the HSX11 gene in C. albicans does not affect the growth of either the yeast or filamentous forms (Leipelt et al., 2001). However, some researchers have suggested that GluCers play important roles in the growth, differentiation and pathogenicity of other fungi. Therefore, to investigate the function of HSX11 in detail, we constructed $h s \times 11$ mutants as described in Methods. For the first allele, HSX11 was replaced with an $h p h 200-U R A 3-h p h 200$ cassette (HSX101) and the remaining allele was replaced with $A R G 4$. The resulting strain was termed HSX102. The URA3 gene was excised from HSX102, generating the Ura-auxotrophic derivative HSX103. Southern blot analysis was performed to confirm that the mutant obtained had a disrupted HSX11 locus (data not shown). Next, to construct the HSX11-recovered strain, a HSX11 expression plasmid (pFLAG-Act1-HSX11) and the vector (pFLAG-ACT1), which contain the URA3 gene as a selectable marker, were introduced into the RP10 locus (Murad et al., 2000) on the chromosome of the hsx11 mutant strain HSX103, yielding strains HSX105 and HSX104, respectively.

\section{Sphingoid base analysis of the mts1 mutants}

To investigate the profile of sphingoid base residues in GluCers of the C. albicans transformants, GluCers were isolated from MTS104 (mts1 mutant), MTS105 (MTS1restored strain) and their corresponding wild-type strain, TUA6. The amounts of GluCers were highly similar among these strains (data not shown). Their sphingoid base compositions were also compared (Fig. 2). The GluCers in the TUA6 strain contained three sphingoid bases: $(E)$-sphing-4-enine, $(E, E)$-sphinga-4,8-dienine and $(E, E)$ 9-methylsphinga-4,8-dienine. In contrast, the GluCers in the MTS104 strain lacked (E,E)-9-methylsphinga-4,8dienine and contained $(E)$-sphing-4-enine and $(E, E)$ sphinga-4,8-dienine. The MTS105 strain recovered the ability to produce (E,E)-9-methylsphinga-4,8-dienine, although the proportion of this sphingoid base in the GluCers was lower than that of TUA6 (wild-type), making $(E, E)$-sphinga-4,8-dienine the most abundant molecule in the GluCers of MTS105. These results indicate that the MTS1 protein (MTS1p) is the only enzyme that introduces a methyl group at the $\mathrm{C} 9$ position of the $\mathrm{LCB}$ of ceramides in C. albicans.

\section{GluCer analysis of hsx11 mutants}

To confirm the absence of GluCers in the $h s \times 11$ disruptant, alkali-stable lipids were isolated from HSX104 (hsx11 mutant), HSX105 (HSX11-restored strain) and the wild-type strain, TUA6. The amounts of GluCers were compared in these strains by TLC (Fig. 3). The HSX105 and TUA6 strains produced similar amounts of GluCers. In contrast, the HSX104 strain completely lacked GluCers. These results show that the hsxl1 mutants are incapable of synthesizing GluCers and that HSX11p is the

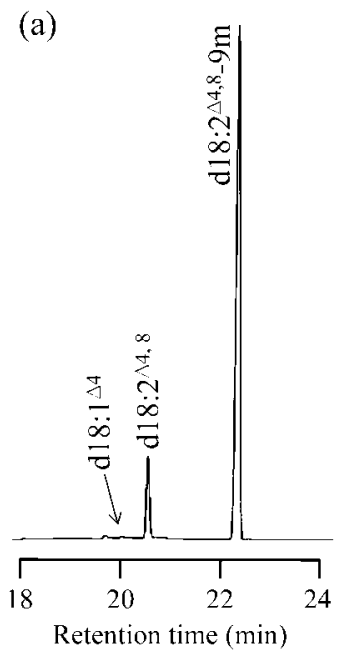

(b)

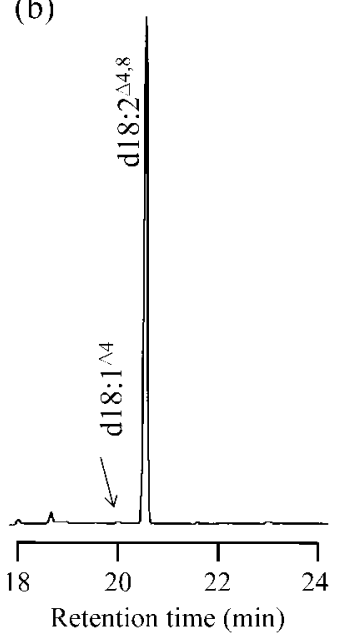

(c)

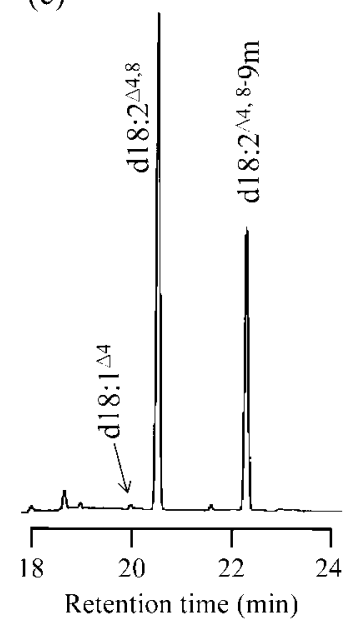

Fig. 2. GLC analysis of fatty aldehydes derived from sphingoid bases. Cells of strains TUA6 (MTS1/MTS1) (a), MTS104 (mts1/ $m t s 1)$ (b) and MTS105 (mts1/mts1 MTS1) (c) were grown overnight at $30{ }^{\circ} \mathrm{C}$. Sphingoid base fractions were subjected to periodate oxidation to obtain fatty aldehydes and then identified by comparing their retention times to known standards prepared from Grifola frondosa sphingoid bases. The abbreviations for sphingoid bases are as follows: $\mathrm{d} 18: 1^{\Delta 4}, 4$ trans-sphingenine; $\mathrm{d} 18: 2^{\Delta 4,8}, 4$-trans-, 8 -transsphingadienine; d18:2 $2^{\Delta 4,8}-9 \mathrm{~m}, \quad$ 9-methyl-,4trans-,8-trans-sphingadienine. 




Fig. 3. TLC analysis of alkali-stable lipids. The alkali-stable lipids extracted from lyophilized cells of TUA6 (HSX11/HSX11) (lane 2), HSX104 (hsx11/hsx11) (lane 3) and HSX105 (hsx11/ $h s \times 11+H S X 11$ ) (lane 4) were dissolved in chloroform/methanol $(1: 1, v / v)$, spotted on a silica gel 60 TLC plate, and the chromatogram was developed using chloroform/methanol/water ( $65: 16: 2$, by vol.) as mobile phase. After development, the plates were sprayed with orcinol/sulfuric acid reagent and heated on a hotplate to detect the GluCers. GluCers prepared from Grifola frondosa were used as standards (lane 1).

only enzyme involved in synthesis of GluCers in $C$. albicans, findings consistent with a previous report (Leipelt et al., 2001).

\section{Growth and morphogenesis of the $C$. albicans gene disruptants}

The (E,E)-9-methylsphinga-4,8-dienine in GluCers is thought to be essential to maintain sufficient membrane fluidity in Kluyveromyces lactis in a low-temperature environment (Tanji et al., 2004). Therefore, to investigate the roles of fungal-specific GluCers during growth at low temperatures, we examined the growth rate of the $m t s 1$ disruptant at $30{ }^{\circ} \mathrm{C}$ and $10{ }^{\circ} \mathrm{C}$ in liquid YPD medium (Fig. 4). There were no significant differences in growth rate between the $m t s 1$ disruptant and the wild-type strain at either temperature. This result indicates that the C9methylation of GluCer is not necessary for the vegetative growth of $C$. albicans at low temperatures.

To investigate the effects of the $m t s 1$ and $h s \times 11$ disruptions on the yeast-to-hypha transition in C. albicans, the genedisrupted strains (MTS104 and HSX104) were incubated in either solid or liquid medium containing $10 \%$ serum. On $10 \%$ serum agar medium at $37{ }^{\circ} \mathrm{C}$, the hyphal growth of both MTS104 and HSX104 was clearly slower than that of wild-type TUA6 (Fig. 5). These phenotypes were restored to wild-type by introducing MTS1 and HSX11 into the $m t s 1$ and $h s \times 11$ mutants, respectively (strains MTS105 and HSX105). On the other hand, there were no significant differences among these $C$. albicans strains in hyphal formation in liquid YPD medium $(\mathrm{pH}$ 7.2) containing $10 \%$ serum (data not shown). These results suggested that not only GluCer synthesis but also the C9-methylation of the LCB play roles in hyphal formation of C. albicans on solid medium.

\section{Susceptibilities of the $m t s 1$ and $h s \times 11$ disruptants to stressors and antifungals}

To investigate the additional effects of the absence of GluCer and C9-methylated GluCer in C. albicans, the mts1 and $h s \times 11$ disruptants were examined for their susceptibilities to a wide range of stress conditions using dilution drop assays (Fig. 6). The stressors high temperature $\left(42^{\circ} \mathrm{C}\right)$, salt $(1 \mathrm{M} \mathrm{NaCl}), 6 \%$ ethanol and $50 \mu \mathrm{g} \mathrm{ml}$ Calcofluor white (CFW) did not affect MTS104 and HSX104 more than TUA6, MTS105 and HSX105. However, MTS104 was more susceptible than TUA6 and MTS105 to the $\beta$-1,3-glucan synthase inhibitor micafungin, while the other antifungals examined did not induce different effects on these strains. On the other hand,

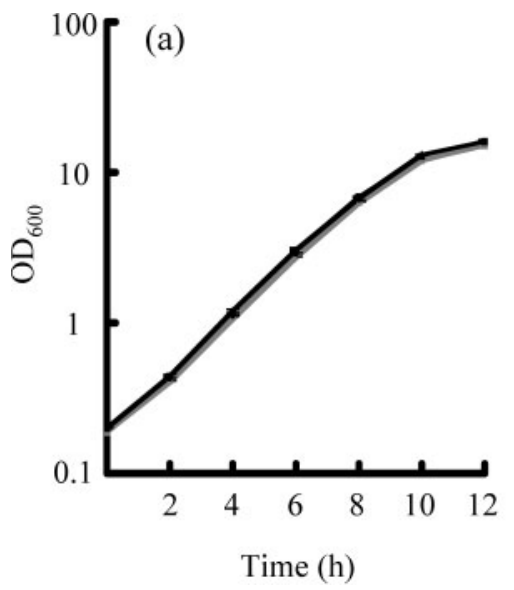

http://mic.sgmjournals.org

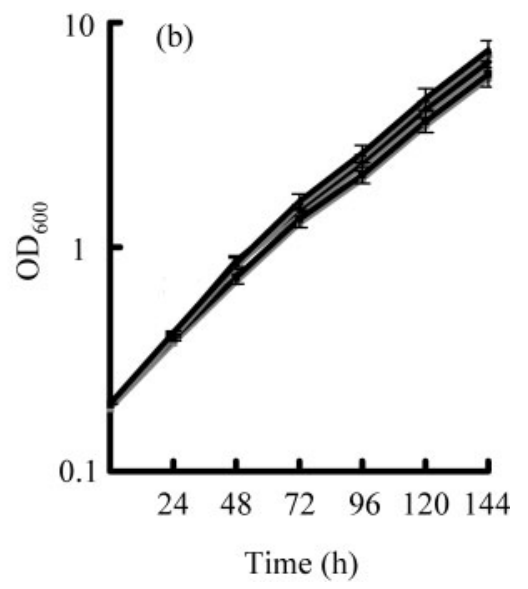

Fig. 4. Growth of $C$. albicans strains in liquid YPD medium. Cells of strains TUA6 (wild-type, -), MTS104 (mts1/mts1, $\mathbf{\Delta})$ and MTS105 (mts1/mts1+MTS1, $\mathbf{\square})$ were pre-cultured overnight at $30{ }^{\circ} \mathrm{C}$ and then inoculated into $50 \mathrm{ml}$ medium and grown to $\mathrm{OD}_{600} 0.2$ on a reciprocal shaker (130 r.p.m.) at $30{ }^{\circ} \mathrm{C}(\mathrm{a})$ and $10^{\circ} \mathrm{C}(\mathrm{b})$. Vertical bars represent the standard errors of three independent experiments. 
Wild-type

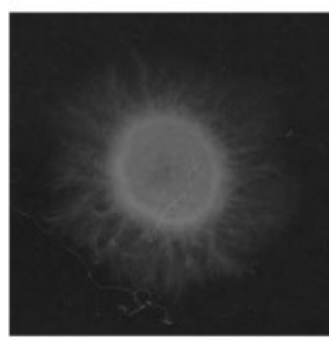

$m t s 1 / m t s 1$

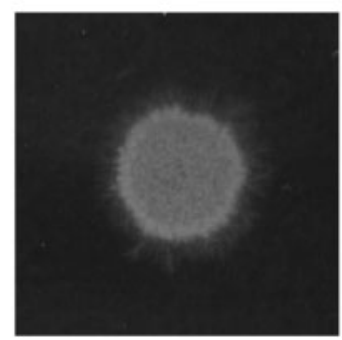

mts $1 / m t s 1$ $+M T S 1$

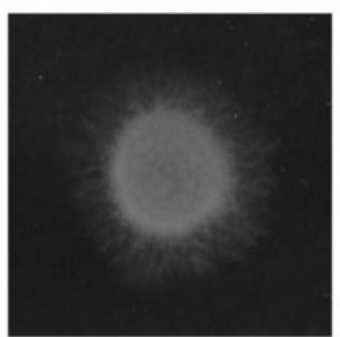

hsx11/hsx11

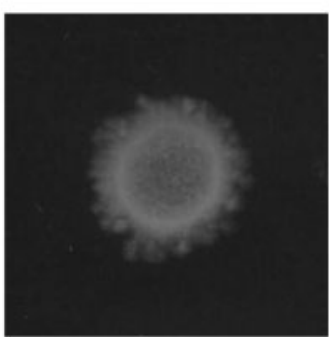

hsx11/hsx11

$+H S X 11$

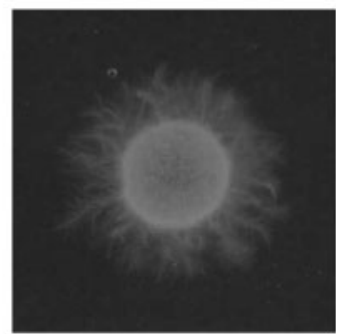

Fig. 5. Colony morphology of C. albicans strains grown on solid agar medium. Cells of strains TUA6 (wild-type), MTS104

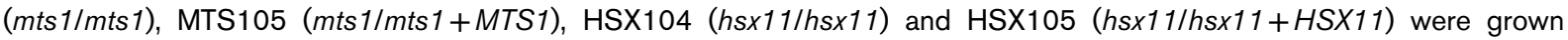
overnight at $30{ }^{\circ} \mathrm{C}$. Then $10^{5}$ cells were spotted onto agar medium containing $10 \%$ serum and grown for 7 days at $37{ }^{\circ} \mathrm{C}$. The results of a representative experiment are shown; four additional independent experiments showed the same results.

HSX104 was more sensitive than TUA6 to $0.05 \%$ SDS, while MTS104 showed no sensitivity to $0.05 \%$ SDS. Moreover, HSX104 was more susceptible than TUA6 and HSX105 to the lanosterol $14 \alpha$-demethylase inhibitor fluconazole, while the other tested antifungals did not induce different effects among these three strains. Thus MTS104 had a different phenotype from HSX104 when treated with SDS and certain antifungals.

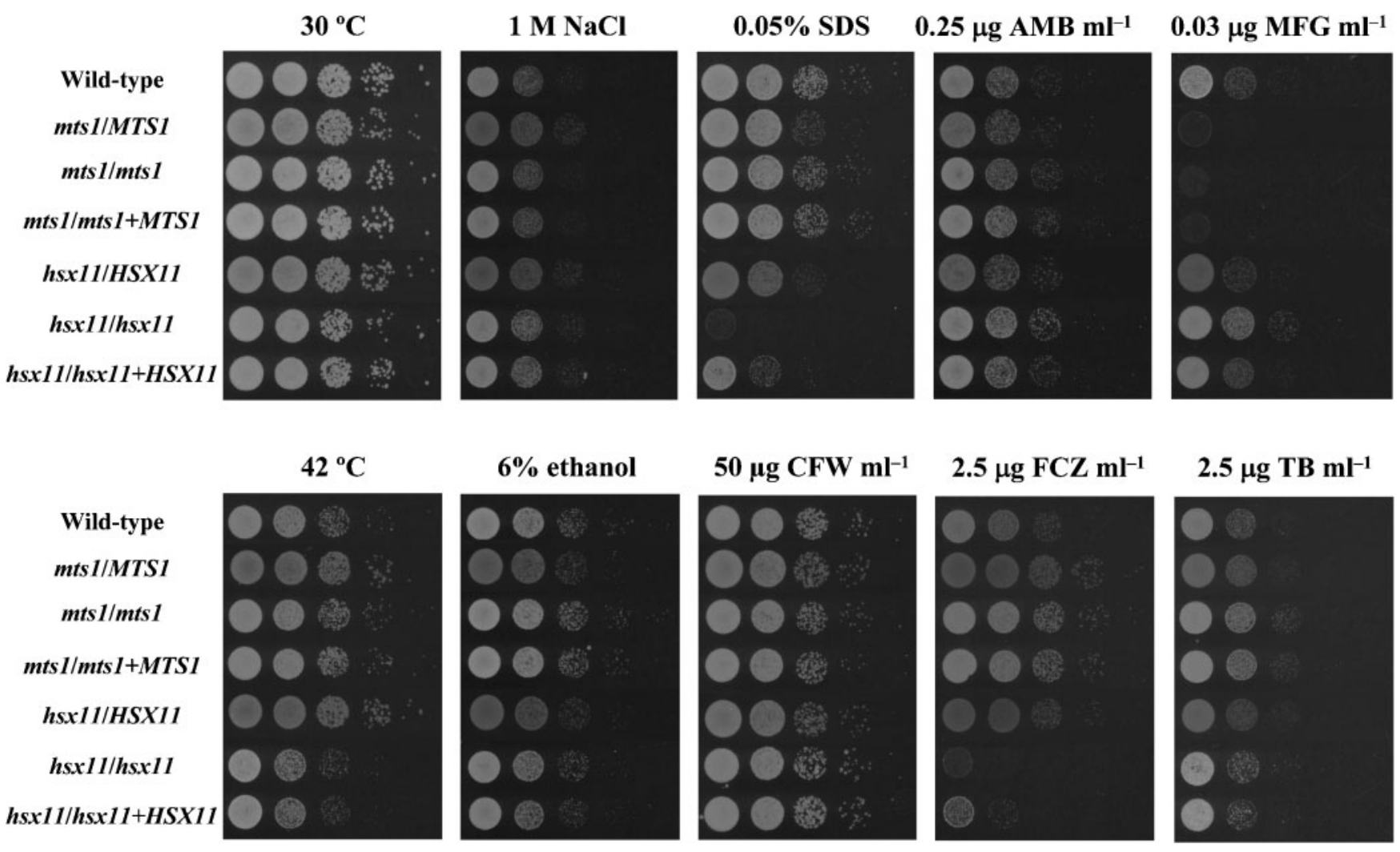

Fig. 6. Susceptibility of C. albicans strains to stressors and antifungals. Cells of strains TUA6 (wild-type), MTS104 ( $m t s 1 /$ $m t s 1), \operatorname{MTS} 105$ (mts1/mts1 + MTS1), HSX104 ( $h s \times 11 / h s \times 11)$ and HSX105 (hsx11/hs $11+H S X 11)$ were grown overnight at $30{ }^{\circ} \mathrm{C}$; then tenfold serial dilutions of the cultures were spotted onto the indicated YPD agar plates and grown for 1 to 2 days at $30{ }^{\circ} \mathrm{C}$. AMB, amphotericin B; CFW, Calcofluor white; FCZ, fluconazole; MFG, micafungin; TB, terbinafine. 


\section{DISCUSSION}

In this study, we characterized the sphingolipid C9methyltransferase gene (MTS1) from C. albicans and constructed a disruptant of this gene by replacing the two MTS1 alleles with two different marker genes. To our knowledge this is the first report of the physiological characterization of a C. albicans mutant with a disrupted sphingolipid C9-methyltransferase gene. We also constructed a disruptant of the HSX11 gene, encoding GluCer synthase, and compared its phenotype to that of the wild-type strain.

Based on the results of the LCB analysis of its GluCers, the $m t s 1$ mutant contained only the sphingoid bases $(E)$ sphing-4-enine and (E,E)-sphinga-4,8-dienine, but not (E,E)-9-methylsphinga-4,8-dienine. The MTS1-restored strain contained all three sphingoid bases $(E)$-sphing-4enine, (E,E)-sphinga-4,8-dienine and (E,E)-9-methylsphinga-4,8-dienine in its GluCers. These results showed that MTS1p is a unique enzyme that introduces a methyl group at the $\mathrm{C} 9$ position of the LCB in ceramides. It was previously shown that a $P$. pastoris strain lacking the sphingolipid $\mathrm{C} 9$-methyltransferase gene produced no (E,E)-9-methylsphinga-4,8-dienine (Ternes et al., 2006), similar to the mts1 mutant of C. albicans. Moreover the databases for Cr. neoformans, Debaryomyces hansenii, Kluyveromyces lactis, Saccharomyces kluyveri and Yarrowia lipolytica indicate that these yeast species have only one sphingolipid C9-methyltransferase gene homologue in their genomes. Therefore, most other yeast species producing GluCers may have only one sphingolipid C9methyltransferase gene. On the other hand, the genomes of both the filamentous ascomycetes A. nidulans and F. graminearum contain two sequences that are homologous to the sphingolipid C9-methyltransferase gene (Ternes et al., 2006). Moreover, it was shown that F. graminearum has two genes with sphingolipid C9-methyltransferase activity (Ramamoorthy et al., 2009).

The amounts of GluCer in the mts1 mutant and wild-type C. albicans strains are highly similar. This indicates that C9-methylation by MTS1p has little influence on subsequent GluCer synthesis. In contrast, disrupting the HSX11 gene in C. albicans leads to the loss of GluCers, indicating that HSX11p is the only enzyme that combines glucose with ceramide for GluCer synthesis. This result is consistent with the first report on hsxll mutants of C. albicans (Leipelt et al., 2001).

The wild-type and $m t s 1$ disruptant strains of C. albicans had the same growth rate at $30{ }^{\circ} \mathrm{C}$ in liquid YPD medium. A $P$. pastoris strain lacking the sphingolipid C9-methyltransferase gene did not have impaired growth compared to the wild-type strain, whereas the $F$. graminearum $\Delta \mathrm{Fg} m t 2$ mutant exhibited severe growth defects (Ternes et al., 2006; Ramamoorthy et al., 2009). It therefore appears that sphingolipid C9-methyltransferase is not necessary for the vegetative growth of yeast but is necessary for the growth of filamentous fungi. Previously, (E,E)-9-methyl- sphinga-4,8-dienine in the GluCers of $K$. lactis was assumed to be essential to maintain sufficient membrane fluidity in a low-temperature environment (Tanji et al., 2004). However, the authors neither constructed any sphingolipid C9-methyltransferase gene mutant of K. lactis nor investigated the growth of any gene mutants of the yeast under low-temperature conditions. In our work, the $m t s 1$ mutant of $C$. albicans had the same growth rate as the wild-type strain, indicating that C9-methylated GluCer is not necessary for the yeast growth of this fungus at $10{ }^{\circ} \mathrm{C}$ or $30{ }^{\circ} \mathrm{C}$. Although the mechanisms that fungi use to adapt to low-temperature stress are complex and many factors are thought to affect the stress response, the loss of C9methylated GluCers is suggested to not influence the yeastlike growth of fungi at low temperature.

When the $m t s 1$ and $h s \times 11$ disruptants were grown in liquid medium to induce the hyphal form, they were able to elongate and produce their germ tubes normally. However, when grown on hyphal-inducing solid media, both the $m t s 1$ and $h s \times 11$ disruptants showed slower elongation than the wild-type. In addition, in our previous report, a sld1 mutant of $C$. albicans, which is unable to produce not only GluCers including (E,E)-sphinga-4,8-dienine as a sphingoid base residue ( $\Delta^{8}$-desaturated GluCer) but also C9methylated GluCers, showed slower elongation than the wild-type, similar to our $m t s 1$ and $h s \times 11$ disruptants. These results suggest that GluCers, including C9-methylated LCB, are at least necessary for normal hyphal elongation of $C$. albicans on solid media. Filamentous growth of $C$. albicans is influenced by the physical environment. Several other $C$. albicans genes needed for filamentous growth on solid medium have been reported (reviewed by Kumamoto \& Vinces, 2005). Thus, several other C9-methylated GluCers also may be involved in the induction of the filamentous form of this fungus on solid media. Some GlyCers in fungi and plants are thought to be highly active in inducing fruiting body formation in Schizophyllum commune (Kawai \& Ikeda, 1985; Kawai et al., 1986). A structural analysis of these GlyCers revealed that C9-methylation in the sphingoid base moiety of fungal GlyCer is essential, while the sugar moiety has no effect. Moreover, the $\Delta \mathrm{Fg} m+2$ mutant of F. graminearum, which has been shown to have reduced amounts of C9-methylated GluCers compared to the wild-type, was defective in mycelial growth (Ramamoorthy et al., 2009). These results and our findings suggest that C9-methylated GluCers may be active in inducing hyphal morphogenesis of C. albicans.

The C. albicans hsx11 disruptant was more sensitive to SDS than the wild-type, but the cell wall stress inducer CFW did not induce different growth effects on the $h s \times 11$ disruptant or wild-type. In addition, the C. albicans hsx 11 disruptant was more susceptible than the wild-type to the lanosterol $14 \alpha$-demethylase inhibitor fluconazole. Fluconazole disrupts cell membrane structures by inhibiting the synthesis of ergosterol, which is a major membrane component. Our data suggest that the loss of GluCer leads to the disruption of cell membrane integrity in C. albicans. Moreover, the 
sphingolipids that are enriched in detergent-resistant membrane microdomains, also known as lipid rafts, are known to be important for normal hyphal growth in C. albicans (Martin \& Konopka, 2004). Martin \& Konopka (2004) showed that myriocin, a specific inhibitor of serine palmitoyltransferase, which catalyses the first step of sphingolipid biosynthesis, affects hyphal elongation in C. albicans. Lipid rafts have been implicated in numerous cellular processes, including signal transduction, protein and lipid sorting, cellular entry of toxins and viruses, and viral budding (reviewed by Chazal \& Gerlier, 2003; Bollinger et al., 2005). Moreover, an antimicrobial peptide inhibitor of the fungal plasma membrane ATPase (Pmalp), which is known to be associated with lipid rafts, blocks the azole resistance of C. albicans (Monk et al., 2005). GluCer is a major component of lipid rafts in most fungi. Therefore, the C. albicans $h s \times 11$ disruptant may be sensitive to SDS and fluconazole due to defects in lipid rafts and thereby show abnormal hyphal growth in response to these agents. Future experiments should examine the lipid composition of membrane detergent-resistant microdomains and the membrane fluidity of the C. albicans hsx 11 mutant in detail.

On the other hand, the C. albicans mts1 disruptant had susceptibility to SDS and fluconazole similar to the wildtype strain, although unlike the $h s x 11$ mutant it was more susceptible than the wild-type to the $\beta$-1,3-glucan synthase inhibitor micafungin. These results indicate that the membrane structure of the C. albicans mtsl disruptant is not disturbed, suggesting that the abnormal hyphal growth of this disruptant is different from those of other GluCer synthesis gene disruptants. Micafungin disrupts the fungal cell wall structure by inhibiting cell wall synthesis. Although the detailed mechanisms of this process are still unclear, deleting the MTS1 gene may affect fungal cell wall synthesis during hyphal growth.

The micafungin susceptibility of the single $m t s 1$ mutant and the MTS1-restored strain was similar to that of the $m t s 1$ knockout mutant. This result indicates that both alleles of MTS1 are necessary for resistance to micafungin. On the other hand, the single disruptant of the HSX11 gene showed SDS and fluconazole resistance similar to the wildtype while the HSX11-restored strain had high susceptibility to SDS and fluconazole, like the $h s \times 11$ knockout strain. In this study, we used the constitutive ACT1 promoter instead of the native HSX11 promoter to restore the expression of the HSX1 gene in the knockout strain. The difference in susceptibility to SDS and fluconazole between the single $h s \times 11$ disruptant and the HSX11-restored strain may be due to the difference in the promoters used for the expression of the HSX11 gene.

The findings of this study suggest that C9-methylated GluCer not only plays functional roles in hyphal morphogenesis but also contributes to cell wall synthesis in $C$. albicans. We have clarified the genes related to the biosynthetic pathway of GluCer in C. albicans. In contrast, the entire metabolic pathway of GluCer, especially its degradation, remains unclear because no fungal genes for GluCer degradation, such as a glucosylceramidase gene, have been identified. To further understand the physiological function of GluCer, it will be necessary to analyse the degradation pathway of GluCer.

\section{ACKNOWLEDGEMENTS}

We thank Dr Mikio Kinoshita and Dr Masao Ohnishi (Obihiro University of Agriculture and Veterinary Medicine) for their technical advice concerning the lipid analysis.

\section{REFERENCES}

Ausubel, F. M., Brent, R., Kingston, R. E., Moore, D. D., Seidman, J. G., Smith, J. A. \& Struhl, K. (1992). Current Protocols in Molecular Biology. New York: Green Publishing Associates \& Wiley-Interscience. Bollinger, C. R., Teichgräber, V. \& Gulbins, E. (2005). Ceramideenriched membrane domains. Biochim Biophys Acta 1746, 284-294.

Chazal, N. \& Gerlier, D. (2003). Virus entry, assembly, budding, and membrane rafts. Microbiol Mol Biol Rev 67, 226-237.

Hanaoka, N., Umeyama, T., Ueno, K., Ueda, K., Beppu, T., Fugo, H., Uehara, Y. \& Niimi, M. (2005). A putative dual-specific protein phosphatase encoded by YVH1 controls growth, filamentation and virulence in Candida albicans. Microbiology 151, 2223-2232.

Kawai, G. \& Ikeda, Y. (1985). Structure of biologically active and inactive cerebrosides prepared from Schizophyllum commune. J Lipid Res 26, 338-343.

Kawai, G., Ohnishi, M., Fujino, Y. \& Ikeda, Y. (1986). Stimulatory effect of certain plant sphingolipids on fruiting of Schizophyllum commune. J Biol Chem 261, 779-784.

Kumamoto, C. A. \& Vinces, M. D. (2005). Alternative Candida albicans lifestyles: growth on surfaces. Annu Rev Microbiol 59, 113-133.

Leipelt, M., Warnecke, D., Zähringer, U., Ott, C., Müller, F., Hube, B. \& Heinz, E. (2001). Glucosylceramide synthases, a gene family responsible for the biosynthesis of glucosphingolipids in animals, plants, and fungi. J Biol Chem 276, 33621-33629.

Levery, S. B., Momany, M., Lindsey, R., Toledo, M. S., Shayman, J. A., Fuller, M., Brooks, K., Doong, R. L., Straus, A. H. \& Takahashi, H. K. (2002). Disruption of the glucosylceramide biosynthetic pathway in Aspergillus nidulans and Aspergillus fumigatus by inhibitors of UDPGlc:ceramide glucosyltransferase strongly affects spore germination, cell cycle, and hyphal growth. FEBS Lett 525, 59-64.

Martin, S. W. \& Konopka, J. B. (2004). Lipid raft polarization contributes to hyphal growth in Candida albicans. Eukaryot Cell 3, 675-684.

Merrill, A. H., Jr, Schmelz, E. M., Dillehay, D. L., Spiegel, S., Shayman, J. A., Schroeder, J. J., Riley, R. T., Voss, K. A. \& Wang, E. (1997). Sphingolipids - the enigmatic lipid class: biochemistry, physiology, and pathophysiology. Toxicol Appl Pharmacol 142, 208-225.

Monk, B. C., Niimi, K., Lin, S., Knight, A., Kardos, T. B., Cannon, R. D., Parshot, R., King, A., Lun, D. \& Harding, D. R. (2005). Surface-active fungicidal D-peptide inhibitors of the plasma membrane proton pump that block azole resistance. Antimicrob Agents Chemother 49, 57-70.

Murad, A. M., Lee, P. R., Broadbent, I. D., Barelle, C. J. \& Brown, A. J. (2000). CIp10, an efficient and convenient integrating vector for Candida albicans. Yeast 16, 325-327. 
Oura, T. \& Kajiwara, S. (2008). Disruption of the sphingolipid $\Delta^{8}$ desaturase gene causes a delay in the morphological change of Candida albicans. Microbiology 154, 3795-3803.

Pinto, M. R., Rodrigues, M. L., Travassos, L. R., Haido, R. M., Wait, R. \& Barreto-Bergter, E. (2002). Characterization of glucosylceramides in Pseudallescheria boydii and their involvement in fungal differentiation. Glycobiology 12, 251-260.

Ramamoorthy, V., Cahoon, E. B., Li, J., Thokala, M., Minto, R. E. \& Shah, D. M. (2007). Glucosylceramide synthase is essential for alfalfa defensin-mediated growth inhibition but not for pathogenicity of Fusarium graminearum. Mol Microbiol 66, 771-786.

Ramamoorthy, V., Cahoon, E. B., Thokala, M., Kaur, J., Li, J. \& Shah, D. M. (2009). Sphingolipid C-9 methyltransferases are important for growth and virulence but not for sensitivity to antifungal plant defensins in Fusarium graminearum. Eukaryot Cell 8, 217-229.

Rittershaus, P. C., Kechichian, T. B., Allegood, J. C., Merrill, A. H., Jr, Hennig, M., Luberto, C. \& Del Poeta, M. (2006). Glucosylceramide synthase is an essential regulator of pathogenicity of Cryptococcus neoformans. J Clin Invest 116, 1651-1659.

Rodrigues, M. L., Travassos, L. R., Miranda, K. R., Franzen, A. J., Rozental, S., de Souza, W., Alviano, C. S. \& Barreto-Bergter, E. (2000). Human antibodies against a purified glucosylceramide from Cryptococcus neoformans inhibit cell budding and fungal growth. Infect Immun 68, 7049-7060.

Saito, M., Saito, M., Cooper, T. B. \& Vadasz, C. (2005). Ethanol-induced changes in the content of triglycerides, ceramides, and glucosylceramides in cultured neurons. Alcohol Clin Exp Res 29, 1374-1383.

Sambrook, J., Fritsch, E. F. \& Maniatis, T. (1989). Molecular Cloning: a Laboratory Manual, 2nd edn. Cold Spring Harbor, NY: Cold Spring Harbor Laboratory.

Sperling, P. \& Heinz, E. (2003). Plant sphingolipids: structural diversity, biosynthesis, first genes and functions. Biochim Biophys Acta $1632,1-15$.
Sperling, P., Zähringer, U. \& Heinz, E. (1998). A sphingolipid desaturase from higher plants. Identification of a new cytochrome $b_{5}$ fusion protein. J Biol Chem 273, 28590-28596.

Takakuwa, N., Kinoshita, M., Oda, Y. \& Ohnishi, M. (2002). Isolation and characterization of the genes encoding $\Delta^{8}$-sphingolipid desaturase from Saccharomyces kluyveri and Kluyveromyces lactis. Curr Microbiol 45, 459-461.

Tanji, M., Kinoshita, M., Yada, H., Yamane, M., Kakuta, Y., Motoshima, H., Oda, Y. \& Ohnishi, M. (2004). Effects of growth temperature on cerebroside content and chemical composition in Kluyveromyces lactis. J Oleo Sci 53, 127-133.

Ternes, P., Franke, S., Zähringer, U., Sperling, P. \& Heinz, E. (2002). Identification and characterization of a sphingolipid $\Delta^{4}$-desaturase family. J Biol Chem 277, 25512-25518.

Ternes, P., Sperling, P., Albrecht, S., Franke, S., Cregg, J. M., Warnecke, D. \& Heinz, E. (2006). Identification of fungal sphingolipid C9-methyltransferases by phylogenetic profiling. J Biol Chem 281, 5582-5592.

Thevissen, K., Warnecke, D. C., François, I. E., Leipelt, M., Heinz, E., Ott, C., Zähringer, U., Thomma, B. P., Ferket, K. K. \& Cammue, B. P. (2004). Defensins from insects and plants interact with fungal glucosylceramides. J Biol Chem 279, 3900-3905.

Umeyama, T., Nagai, Y., Niimi, M. \& Uehara, Y. (2002). Construction of FLAG tagging vectors for Candida albicans. Yeast 19, 611-618.

Umeyama, T., Kaneko, A., Nagai, Y., Hanaoka, N., Tanabe, K., Takano, Y., Niimi, M. \& Uehara, Y. (2005). Candida albicans protein kinase CaHsl1p regulates cell elongation and virulence. Mol Microbiol 55, 381-395.

Warnecke, D. \& Heinz, E. (2003). Recently discovered functions of glucosylceramides in plants and fungi. Cell Mol Life Sci 60, 919941.

Edited by: M. Schweizer 